\title{
Photovoltaic Properties of Thermally-Grown Selenium-Doped Silicon Photodiodes for Infrared Detection Applications
}

\author{
Oday A. HAMMADI* \\ Department of Physics, College of Education, Al-Iraqia University, Baghdad, Iraq \\ *Corresponding author: Oday A. HAMMADIＥmail: odayata2001@yahoo.com
}

\begin{abstract}
In this work, the photovoltaic properties of selenium-doped silicon photodiodes were studied. Influence of illumination of the impurity absorption range on the current-voltage and spectral characteristics of the fabricated device were considered. The photoresponse dependencies on the electric intensity, current, and radiation power at the sample were observed. Results obtained in this work showed that the current-sensitivity of the fabricated structures at the forward bias was rather higher than that of photoresistors. The photosensitivity and detectivity were up to $2.85 \times 10^{-16} \mathrm{~W} \cdot \mathrm{Hz}^{-1 / 2}$ and $2.1 \times 10^{11} \mathrm{~cm} \cdot \mathrm{Hz}^{1 / 2} \mathrm{~W}^{-1}$, respectively.
\end{abstract}

Keywords: Silicon devices, selenium dopant, photodiodes, photovoltaic properties

Citation: Oday A. HAMMADI, "Photovoltaic Properties of Thermally-Grown Selenium-Doped Silicon Photodiodes for Infrared Detection Applications," Photonic Sensors, 2015, 5(2): 152-158.

\section{Introduction}

The interest in the development of extrinsic photodetectors has been increased by the possibility of fabricating monolithic thermal imaging systems, photodetector arrays, charge-coupled devices, etc., based on extrinsic silicon. The fact that the silicon integrated-circuit technology is highly developed facilitates the development of these devices [1-4].

The photoconducting properties of seleniumdoped silicon were first investigated in the mid 1970's. The early work was characterized by low selenium concentrations [5] and high compensation ratios [6] which led to inferior detector performance. Selenium is an n-type impurity and has low boiling point temperature $\left(690^{\circ} \mathrm{C}-700^{\circ} \mathrm{C}\right)$ and diffuses in silicon fast. These factors complicate the doping of silicon during silicon crystal growth, which occurs at about $1410^{\circ} \mathrm{C}$, but permit doping after crystal growth by the closed quartz tube. The variables in closed tube diffusion are the diffusion temperature, the quench procedure, the diffusion time, and the diffusion vapor pressure [7].

Quenching is a useful technique to be employed for impurities whose equilibrium solubilities vary with temperature. Quenching from elevated temperatures is carried in short time compared with the time required to establish equilibrium under this circumstance. Studies related to detector optimization were carried out for silicon-selenium $(\mathrm{Si} / \mathrm{Se})$ as a part of near infrared (NIR) silicon detector development. The parameters determining the carrier distribution at the non-equilibrium condition in the base region of structures made on the base of semiconductors compensated with deep levels depend on the current. Such distribution usually leads to the formation of the negative resistance region on the $I-V$ characteristics curve [8].

Received: 16 January 2015 / Revised version: 6 Febrary 2015

(C) The Author(s) 2015. This article is published with open access at Springerlink.com

DOI: $10.1007 / \mathrm{s} 13320-015-0241-4$

Article type: Regular 
The high photosensitivity of the Si p-n structure has been presented in the previous work [9-12].

In the present paper, the results of $3 \mu \mathrm{m}-5 \mu \mathrm{m}$ illumination of the selenium-doped silicon p-n structure are presented. It is shown that effective injection amplification might be realized in the case of illumination from the impurity range. The photodiode properties have been compared with silicon-selenium $(\mathrm{Si} / \mathrm{Se})$ photoresistors of the same geometrical sizes.

\section{Experiment}

Substrates of (111) p-type boron-doped silicon were doped by selenium using the thermal growth method at the temperature of $1100{ }^{\circ} \mathrm{C}$ in an evacuated quartz-tube. The concentration of residual atoms in initial silicon was determined to be $10^{11} \mathrm{~cm}^{-2}-10^{12} \mathrm{~cm}^{-2}$. These samples were then etched for one minute in an $\mathrm{HNO}_{3}: \mathrm{HF}: \mathrm{H}_{2} \mathrm{O}$ solution of 20:5:6 ratios. The concentration of electro-active selenium atoms in silicon after the doping process was $6 \times 10^{16} \mathrm{~cm}^{-3}$, which was determined by the secondary ion mass spectrometry (SIMS). The maximum photoresponse at the temperature of $263 \mathrm{~K}$ was observed at the wavelength of $3.8 \mu \mathrm{m}$, and the long-wave boundary level corresponding to $50 \%$ relative spectral characteristics was observed at $4.2 \mu \mathrm{m}$. The optical-ionization energy level of selenium was about $0.301 \mathrm{eV}$.

The photodiode had a tablet form of $2 \mathrm{~mm}$ in diameter and $0.5 \mathrm{~mm}$ in thickness on which contacts were made. Figure 1 shows the scanning electron microscope (SEM) image of the Se-doped silicon photodiode prepared in this work. Highly-pure gold and aluminum powder were used to make the Ohmic and rectifying contacts, respectively. The illumination was employed to the $p-n$ junction side from a looking-glass monochromator. The prepared samples were then transferred to the freezing system in order to employ the low-temperature condition.

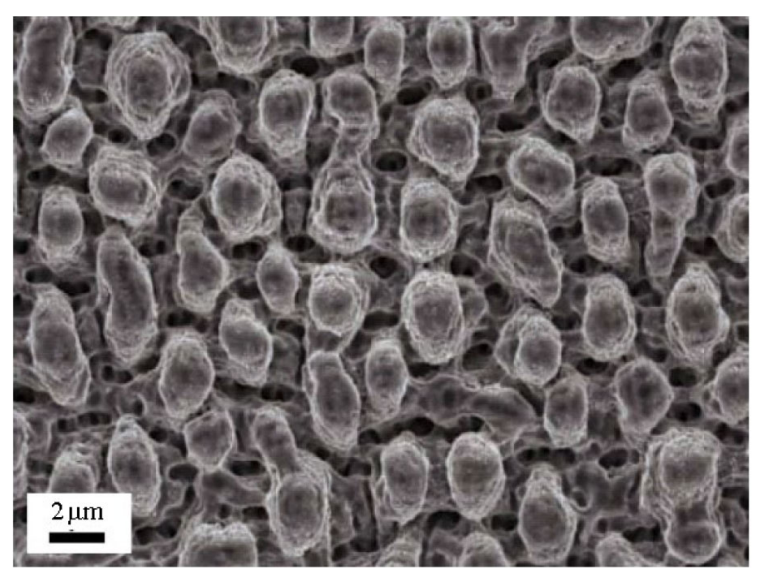

Fig. 1 SEM image of the Se-doped silicon substrate.

A silicon filter was used to determine the illumination characteristics. The time characteristics were measured by frequency-dependent photoresponse. For this purpose, the mechanical modulation with a disk was employed, whose rotation velocity was controlled by changing the feeding voltage. The measurements were carried out in the generator voltage region.

\section{Results and discussion}

Incorporation of selenium dopants in silicon substrates leads to narrowing the energy band gap of the Se:Si structure, and hence their cutoff wavelength is increased beyond that of the intrinsic silicon $(1.1 \mu \mathrm{m})$.

The $I-V$ characteristics of the prepared samples were recorded at different illumination levels, $\phi=0$ (corresponding to no-illumination condition), $\phi_{1}=$ $40 \mathrm{~mW} / \mathrm{cm}^{-2}, \quad \phi_{2}=90 \mathrm{~mW} / \mathrm{cm}^{-2}$, and $\phi_{3}=$ $200 \mathrm{~mW} / \mathrm{cm}^{-2}$. At $T=300 \mathrm{~K}$, these characteristics had a typical low-power rectifier form. As shown in Fig. 2, a negative resistance region on $I-V$ characteristics was formed in the forward direction while an Ohmic region was observed without illumination or at low illumination level. Increasing the applied voltage beyond $5 \mathrm{~V}$, the region changed into a $J \approx V^{3 / 2}$ region, which again changed into a linear behavior at large illumination levels after which impact ionization began. 


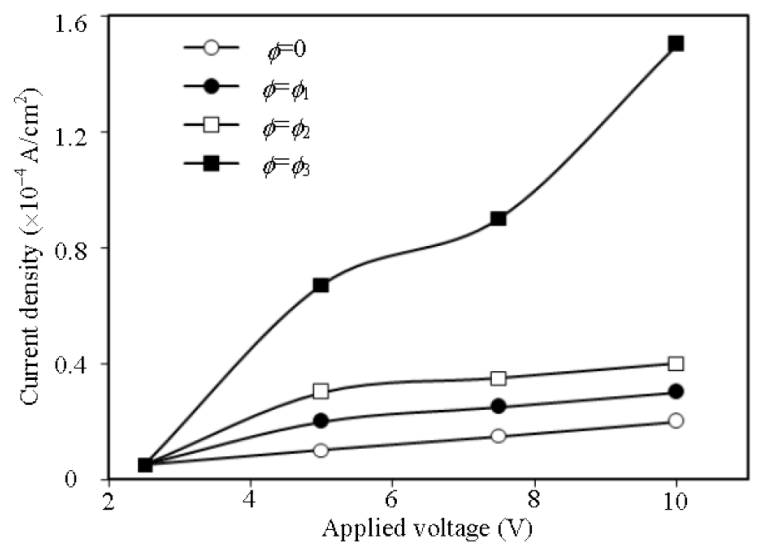

Fig. 2 Illumination-dependent $I-V$ characteristics of Se-doped silicon photodiode structures at different injection levels $\left(\phi=0, \phi_{1}=40 \mathrm{~mW} / \mathrm{cm}^{2}, \phi_{2}=90 \mathrm{~mW} / \mathrm{cm}^{2}\right.$ and $\phi_{3}=$ $\left.200 \mathrm{~mW} / \mathrm{cm}^{2}\right)$.

For most diodes, a region on the $I-V$ characteristics - where the voltage did not depend on the current - was observed. With initial illumination, the breakdown in the $I-V$ characteristics was highly deformed, and the small Ohmic region changed into a slow increasing-current behavior through which a sub-linear dependence on the voltage was observed. Then this behavior changed into linear dependence. Figure 3 indicates the behavior of sensitivity $(R)$ of the prepared photodiode as a function of the incident wavelength. It is clear that the sensitivity was maximized at the wavelength region of $3.5 \mu \mathrm{m}-$ $3.8 \mu \mathrm{m}$, which could be considered as the operation spectral region of such photodiodes.

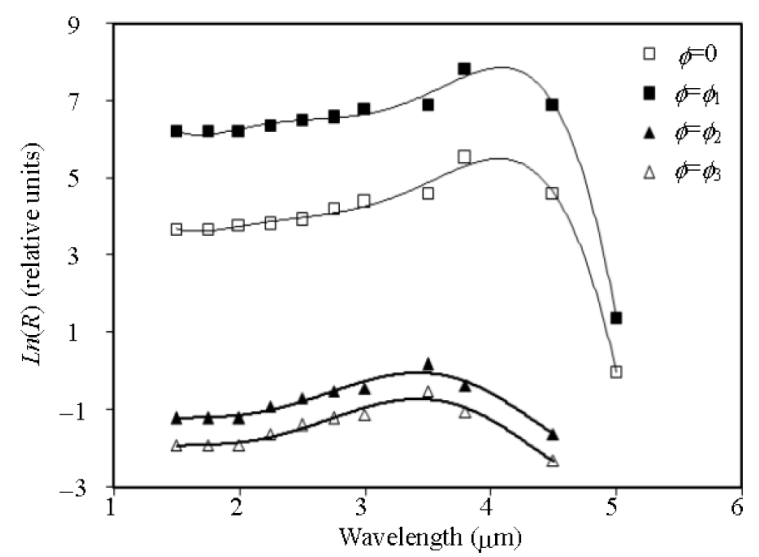

Fig. 3 Variation of spectral characteristics with the incident wavelength on the fabricated photodiodes (upper) and equivalent photoresistors (lower).
Figures 4 and 5 indicate the dependencies of injection amplification $\left(K_{\mathrm{IA}}\right)$ and conductivity modulation $\left(K_{m}\right)$, respectively, upon the electric field applied to the device. The applied voltage and electric field intensity were corresponding as the distance along which the voltage applied was approximately constant $(100 \mu \mathrm{m})$. Dependencies of current sensitivities and dark current densities upon the electric field intensities for the photodiode increased fast below $300 \mathrm{~V} / \mathrm{cm}$, showed an approximately constant behavior beyond $300 \mathrm{~V} / \mathrm{cm}$ and kept this stability below $10^{3} \mathrm{~V} / \mathrm{cm}$.

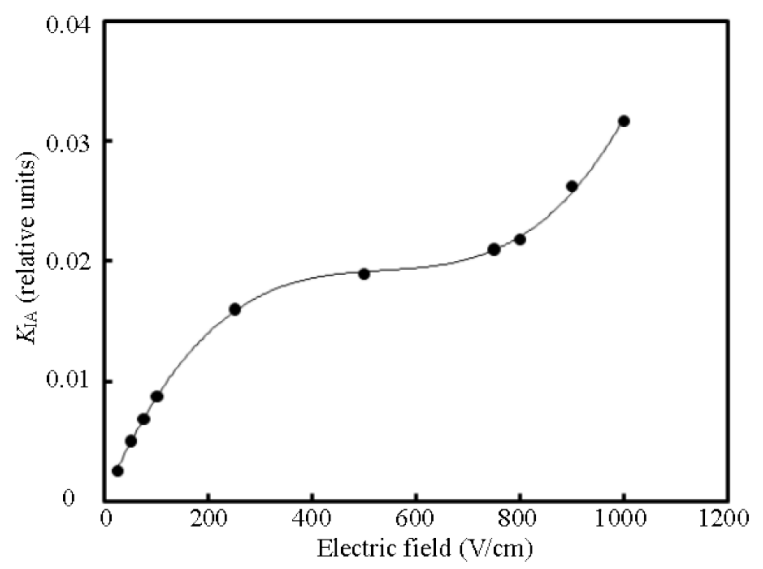

Fig. 4 Dependency of injection amplification on the applied electric field.

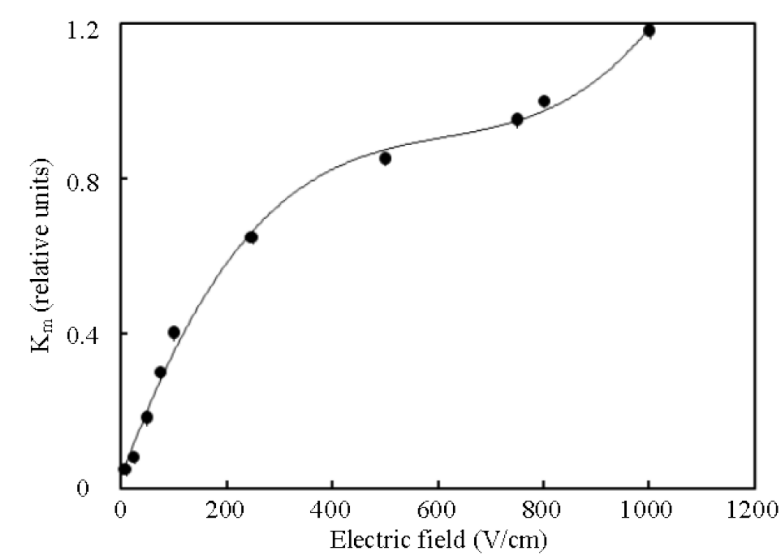

Fig. 5 Dependency of conductivity modulation on the applied electric field.

At lower field intensities $(50 \mathrm{~V} / \mathrm{cm})$, the samples had a sensitivity of $20 \mathrm{~A} / \mathrm{W}$. Both dependencies increased linearly beyond the field intensity of $10^{3} \mathrm{~V} / \mathrm{cm}$. The identical behaviors described above indicated that the bulk distribution of the fabricated 
structure was homogeneous over the whole range of the applied electric field [13-15].

If the noise current density and frequency bandwidth are taken into account, the value of the sensitivity at which the signal recorded without illumination is up to $10^{-11} \mathrm{~A} / \mathrm{Hz}^{1 / 2}$, and then the threshold sensitivity $\left(P_{\text {min }}\right)$ is calculated as [16]

$$
P_{\min }=\frac{R}{I_{n}} \sqrt{\Delta f} \approx 5 \times 10^{-13} \quad\left(\mathrm{~W} \cdot \mathrm{Hz}^{\frac{1}{2}}\right)
$$

where $I_{n}$ is the noise current (A), $R$ is the sensitivity $(\mathrm{A} / \mathrm{W})$, and $f$ is the frequency bandwidth $(\mathrm{Hz})$.

The curves in Figs. 6 and 7 correspond to each other, which makes an evidence of the amplification mechanisms. A sharp sensitivity growth of particular samples was observed at the distribution point, and the sensitivity increased for several times in this region, as shown in Fig. 6. In principle, this behavior is expected to continue beyond the maximum value of the applied electric field. However, many limitations may exist to stop this behavior, and the most important one is the saturation effect.

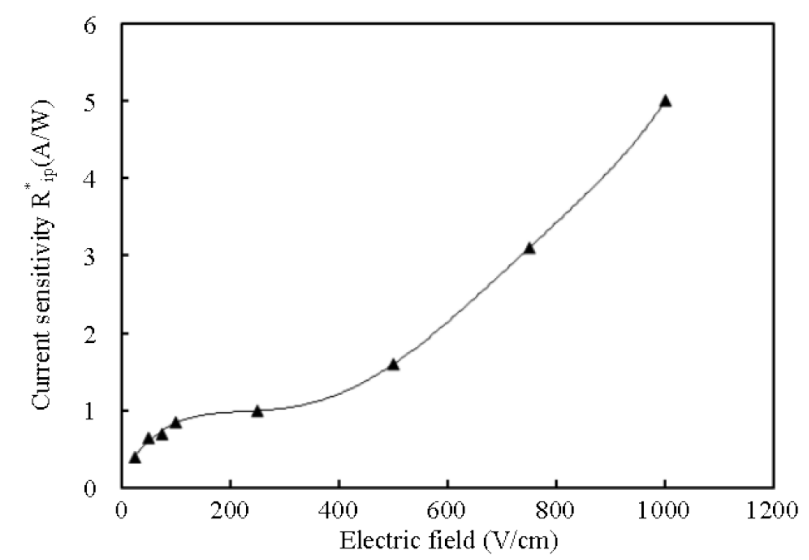

Fig. 6 Variation of the current sensitivity with the applied electric field for the fabricated photodiodes.

Degradation in the bulk may be expected at extremely higher values of the applied electric field along the geometry of the fabricated structure [17].

One may consider the values of dark current densities are high (up to $5 \mathrm{~A} / \mathrm{cm}^{2}$ ) when compared to similar structures fabricated by some other methods and techniques [18, 19]. However, these values are acceptable to a reasonable degree especially when such structures are used in thermal detection applications [20-23].

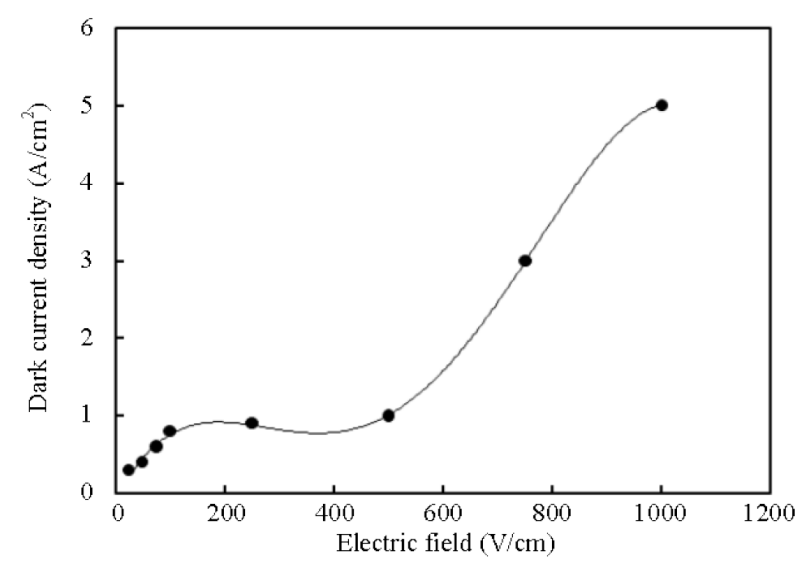

Fig. 7 Variation of the dark current density with the applied electric field for the fabricated photodiodes.

Most photodiodes have a high photosensitivity $(1560 \mathrm{~A} / \mathrm{W})$ at the wavelength of $3.8 \mu \mathrm{m}$ near the breakdown point, as shown in Fig. 3, corresponding to the quantum efficiency of 300 electron/hole. The noise in a single band is about $10^{-11} \mathrm{~A} / \mathrm{Hz}^{1 / 2}$, and hence the threshold sensitivity $\left(P_{\min }\right)$ is about $5 \times 10^{-15} \mathrm{~W} / \mathrm{Hz}^{1 / 2}$.

Figure 8 shows the variation of the current sensitivity with the applied electric field. As shown, a fast increase in the current sensitivity was observed before applying an electric field of about $200 \mathrm{~V} / \mathrm{cm}$ while it became relatively constant during the range of $200 \mathrm{~V} / \mathrm{cm}-600 \mathrm{~V} / \mathrm{cm}$ and increased again with a slow rate beyond $600 \mathrm{~V} / \mathrm{cm}$. Below $200 \mathrm{~V} / \mathrm{cm}$, the photodiode may efficiently be used in the applications requiring fast-response photodetectors. In the second region on the curve, the nearly constant sensitivity is not preferred in detection applications but required in some uses of photodiodes, such as photo-electronic devices employed in broadband infrared absorption.

Similarly, Fig. 9 shows the variation of the dark current density with the applied electric field, and the same behavior was observed below $200 \mathrm{~V} / \mathrm{cm}$. Then the dark current density tended to be constant within $75 \mathrm{~A} / \mathrm{cm}^{2}-90 \mathrm{~A} / \mathrm{cm}^{2}$ during the range of $200 \mathrm{~V} / \mathrm{cm}-800 \mathrm{~V} / \mathrm{cm}$. Such a behavior was preferred to recognize the characteristics of the 
fabricated photodiodes in the same range of the applied electric field considered for the optimum operation. Similarly, the dark current density began to increase beyond the applied electric field of $10^{3} \mathrm{~V} / \mathrm{cm}$.

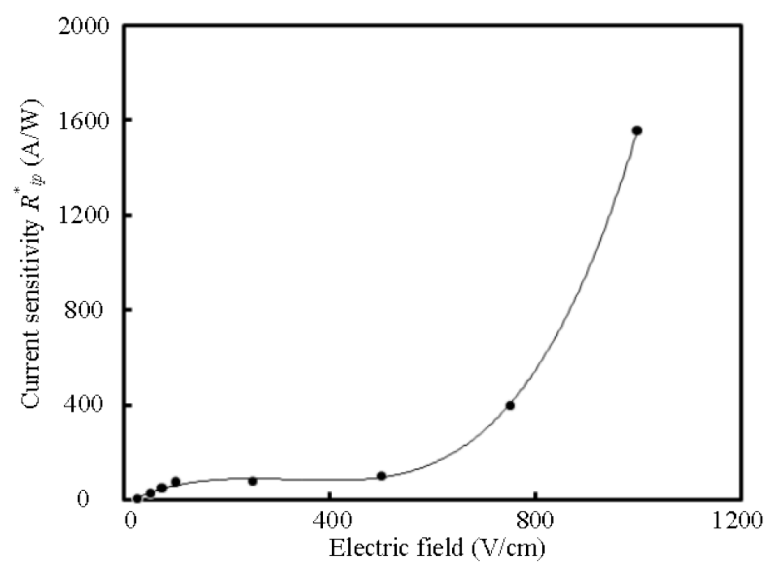

Fig. 8 Variation of the current sensitivity with the applied electric field for the fabricated photoresistors.

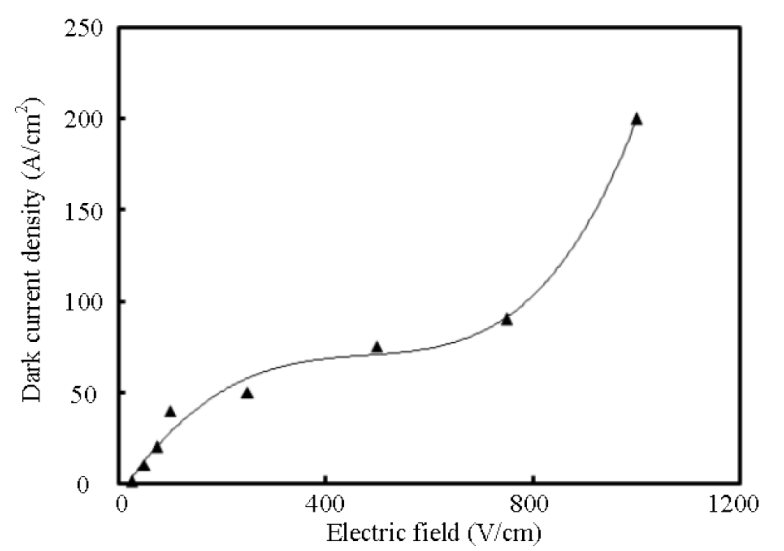

Fig. 9 Variation of the dark current density with the applied electric field for the fabricated photoresistors.

Radiation of the thermal source $(T=500 \mathrm{~K})$ at the receiver temperature of $263 \mathrm{~K}$ and at the field-of-view (FOV) on the background of $60^{\circ}$ was used to measure the detectivity of the fabricated photodiodes. For the applied field of about $10 \mathrm{~V} / \mathrm{cm}$, the specific detectivity $\left(D^{*}\right)$ was about $8 \times 10^{9} \mathrm{~cm} \cdot \mathrm{Hz}^{1 / 2} / \mathrm{W}$. Practically, at the electric fields of $500 \mathrm{~V} / \mathrm{cm}-600 \mathrm{~V} / \mathrm{cm}$, the specific detectivity was not changed, although such a sensitivity increased abruptly, but in that case the noise-level increased as well.

An idealized curve of the relative spectral sensitivity with a peak wavelength of $3.8 \mu \mathrm{m}$ was used to define the absolute spectral detectivity. Further, $D^{*}{ }_{\max } / D^{*}{ }_{500}$ was calculated to be 12.5 in this case, and $D^{*}{ }_{\text {max }} \approx 10^{11} \mathrm{~cm} \cdot \mathrm{Hz}^{1 / 2} / \mathrm{W}$ at the FOV of $60^{\circ}$.

The temperature-dependent behaviors of the sensitivity and detectivity were observed within the range of $110 \mathrm{~K}-150 \mathrm{~K}$, as shown in Figs. 10 and 11. It is evident that the optimum operation of photodiodes in this region of the electromagnetic spectrum $(3 \mu \mathrm{m}-5 \mu \mathrm{m})$ could be obtained effectively at relatively low temperatures. This imposes attempting to reduce temperatures much more in order to introduce the characteristics of such devices at lower temperatures.

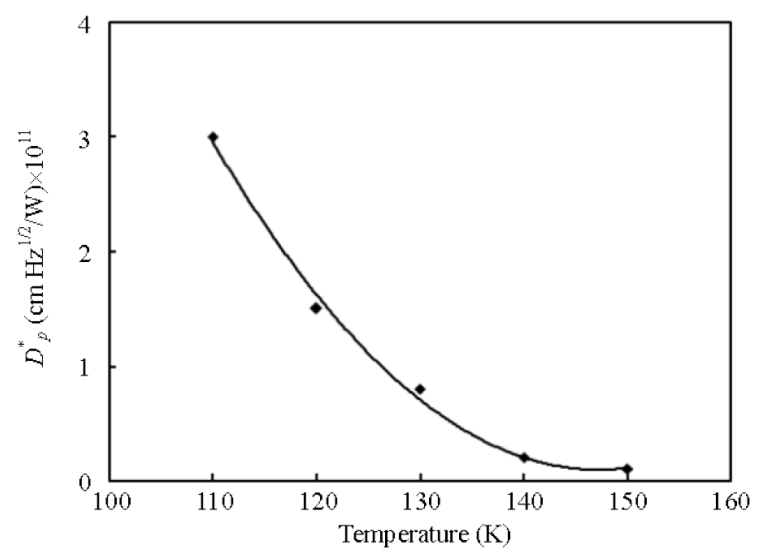

Fig. 10 Variation of the detectivity with the temperature for the fabricated Se-doped silicon photodiode.

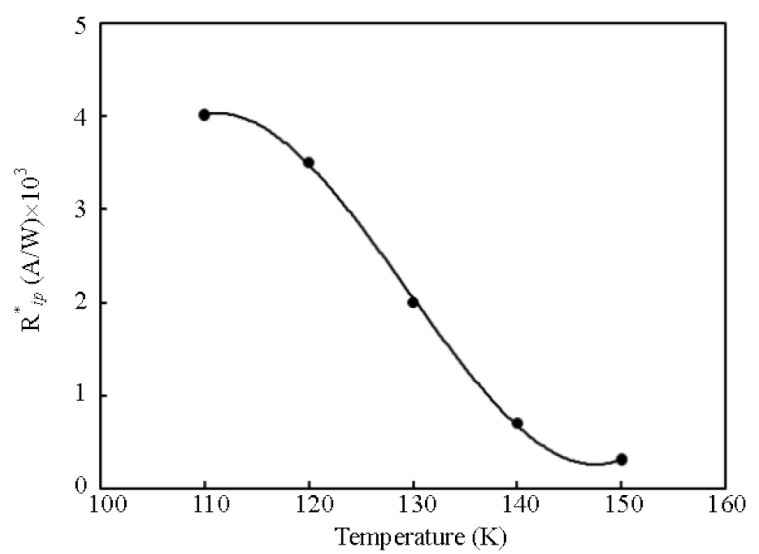

Fig. 11 Variation of the current sensitivity with the temperature for the fabricated Se-doped silicon photodiode.

Cooling is important when using the fabricated structures in infrared detection applications in order to obtain the optimum characteristics of detection 
(sensitivity and detectivity). Accordingly, the higher detectivity is expected at lower temperatures of the Se-doped silicon photodiodes (below $110 \mathrm{~K}$ ). However, the sensitivity shows the saturation $(\sim 4000 \mathrm{~A} / \mathrm{W})$ at temperatures lower than $110 \mathrm{~K}$. This requires a compromise to reach the maximum values of both the sensitivity and detectivity. Some detection applications require the photodiode to work at the highest detectivity as the sensitivity is constant enough to respond as the detectivity is decreased by an order of magnitude when the photodiode temperature is changed by $25 \mathrm{~K}$ only $(125 \mathrm{~K}-150 \mathrm{~K})$. So efficient cooling (below $-125{ }^{\circ} \mathrm{C}$ ) is important for the optimum operation of such structures in infrared detection applications.

The magnitudes of the sensitivity and detectivity did not depend on the frequency in the range around $200 \mathrm{kHz}$. Increasing the detectivity with decreasing the field-of-view on a background up to $30^{\circ}$ was registered to be $2.1 \times 10^{11} \mathrm{~cm} \cdot \mathrm{Hz}^{1 / 2} / \mathrm{W}$.

\section{Conclusions}

From the results obtained from this work, it can be concluded that most photodiodes had a high photosensitivity near the breakdown point. The detectivity was increased with a decrease in the field-of-view. Selenium-doped silicon diodes could successfully be used as radiation photoreceivers in the atmospheric window of $3 \mu \mathrm{m}-5 \mu \mathrm{m}$ with reasonable efficiencies. Dark current densities of these devices were high when compared to similar structures fabricated by some other methods and techniques.

Open Access This article is distributed under the terms of the Creative Commons Attribution License which permits any use, distribution, and reproduction in any medium, provided the original author(s) and source are credited.

\section{References}

[1] R. A. Ismail, O. A. Abdulrazaq, A. A. Hadi, and O. A. Hamadi, "Characterization of Si p-n photodetectors produced by laser-induced diffusion," International Journal of Modern Physics, 2005, 19(31): 4619-4628.

[2] O. A. Hamadi and K. Z. Yahiya, "Physics: heterojunction detectors optical and electrical properties of selenium-antimony heterojunction formed on silicon substrate," University of Sharjah Journal of Pure and Applied Sciences, 2007, 4(2): $1-11$.

[3] O. A. Hamadi, "Profiling of antimony diffusivity in silicon substrates using laser-induced diffusion technique," Iraqi Journal of Applied Physics Letters, 2010, 3(1): 23-26.

[4] P. A. R. Khan, S. M. Butta, and S. A. Malik, "Modeling of transport properties of amorphous silicon solar cells," Iraqi Journal of Applied Physics Letters, 2010, 6(1): 25-32.

[5] H. R. Vydyanath, J. S. Lorenzo, and F. A. Kroger, "Defect pairing diffusion, and solubility studies in selenium-doped silicon," Journal of Applied Physics, 1978, 49(12): 5928-5937.

[6] H. R. Vydyanath, W. J. Helm, J. S. Lorenzo, and S. T. Hoelke, "Development of selenium-doped silicon for 3-5 $4 \mathrm{~m}$ applications," Infrared Physics, 1979, 19(1): 93-102.

[7] A. A. K. Hadi and O. A. Hamadi, "Optoelectronic characteristics of as-doped silicon photodetectors produced by LID technique," Iraqi Journal of Applied Physics Letters, 2008, 1(2): 23-26.

[8] S. M. Sze, Semiconductor devices physics and technology. Taiwan, China: Murray Hill, 1981: 103-154.

[9] A. T. Maher, B. G. Streetman, and N. J. Holonyak "Infrared detection properties of Zn-doped Si p-i-n diodes," IEEE Transaction on Electron Devices, 1969, 16(11): 963-964.

[10] V. M. Haroutyunyan, Z. N. Adamyan, R. S. Barseghyan, F. V. Gasparyan, M. H. Azaryan, B. O. Semerdjian, et al., "Phenomena in silicon photodiodes doped with Zn and S," Infrared Physics, 1985, 26(5): 267-272.

[11] N. Sclar, "The effect of dopant diffusion vapor pressure on the properties of sulfur and selenium doped silicon infrared detectors," Journal of Applied Physics, 1981, 52(8): 5207-5217.

[12] M. O. Henry, J. D. Campion, K. G. McGuigan, M. L. W. Thewalt, and E. C. Lightowlers, "A photoluminescence study of zinc-implanted silicon," Materials Science and Engineering: B, 1989, 4(1-4) : 201-204.

[13] S. D. Kurmashev, V. I. Stafeev, I. Vikulin, and A. N. Sofronkov, "Injection-amplification IR photodiodes," in Proc. SPIE, vol. 3182, pp. 59-67, 1997.

[14] V. I. Stafeev, "Photodetectors with internal injection amplification," Applied Physics, 2007, 2: 82-85.

[15] I. M. Vikulin, S. D. Kurmashev, and V. I. Stafeev, 
"Injection-based photodetectors," Semiconductors, 2008, 42(1): 112-127.

[16] S. Hu, P. Han, S. Wang, X. Mao, X. Li, and L. Gao, "Structural and optoelectronic properties of selenium-doped silicon formed using picosecond pulsed laser mixing," Physica Status Solidi (A) Applications and Materials Science, 2012, 209(12): 2521-2526.

[17] A. Taleb, K. A. Al-Naimee, S. S. Ahmed, and R. Meucci, "Efficiency enhancement of photovoltaic silicon cell by ultrashort laser pulses," Iraqi Journal of Applied Physics, 2009, 5(2): 33-35.

[18] W. K. Hamoudi and R. O. D. Ali, "Structural characteristics study of indium diffusion in silicon using a pulsed Nd:YAG laser," Iraqi Journal of Applied Physics, 2005, 1(1): 34-38.

[19] J. P. Mailoa, A. J. Akey, C. B. Simmons, D. Hutchinson, J. Mathews, J. T. Sullivan, et al., "Room-temperature sub-band gap optoelectronic response of hyperdoped silicon," Nature Communications, 2014, 5: 1-8.

[20] H. G. Grimmeiss, E. Janzen, and B. Skarstam, "Electronic properties of selenium-doped silicon," Journal of Applied Physics, 1980, 51(7): 3740-3745.

[21] H. R. Vydyanath, J. S. Lorenzo, and F. A. Kröger, "Defect pairing diffusion, and solubility studies in selenium-doped silicon," Journal of Applied Physics, 1978, 49(12): 5928-5937.

[22] L. Gao, P. Han, X. Mao, Y. Fan, S. Hu, C. Zhao, et al., "Deep energy levels formed by $\mathrm{Se}$ implantation in Si," Chinese Physics Letters, 2011, 28(3): 036108-1-036108-2.

[23] Y. A. Astrov, L. M. Portsel, A. N. Lodygin, and V. B. Shuman, "Planar microdischarge device for high-speed infrared thermography: application of selenium-doped silicon detectors," Journal of Applied Physics, 2008, 103(11): 114512-1-114512-6. 